\title{
Benefits from Fruit/Vegetable Consumption on Erectile Dysfunction among Diabetic Albanian Men
}

\author{
Kim M. DRASA Jr. ${ }^{1}$, Bruka L. ${ }^{2}$ \\ Central Polyclinic, Tirana, Albania
}

\begin{abstract}
Aim: The aim of our study was to know the association between ED and the consumption of fruit/vegetables (F/V) among diabetic Albanian men. Materials and Methods: In the survey, the daily consumption of $F / V$ was estimated on the basis of a series of questions: How often do you eat F/V per day? The responses were: "Less than 3/3 to 5/more than 5 times (serving) per day". The association between F/V consumption and ED was examined using logistic regression. Results: 475 diabetic men, average age of 60 years (range, 20-70). 205 cases were identified with ED. 89.1\% having type 2 diabetes, 7.3\% type 1 diabetes and 3.6\% were unclassifiable. $82 \%$ were overweight or obese. $26.8 \%(95 \% C I)$ reported having ED. Higher proportions of patients with ED reported poor/fair health, long durations of diabetes, obesity, physical inactivity, formerly smokers, divorced and not consuming enough $F / V(<2 s e r v i n g)$. An inverse association was found between ED and F/V consumption. The rate of ED decreased by $13 \%$ with each increase of 1 serving consumption of F/V. Conclusion: $E D$ is common among diabetic Albanian men. The occurrence of ED among diabetic men is fairly common. ED is inversely associated significantly with the consumption of $F / V$ in the diabetic populations. Our findings suggest that adopting a healthy diet, healthy lifestyle, reducing or controlling BMI and activities that help consume more F/V would reduce the risk of developing $E D$ and would be beneficent for diabetic men with $E D$.
\end{abstract}

Keywords: diabetic, erectile dysfunction, fruit/vegetable, consumption

\section{Introduction}

Currently, nearly over 287,4 billion people worldwide are living with diabetes mellitus (DM). Over the last decade, the age - standardized prevalence of DM has increased from $3.5 \%$ in $1998 / 1999$ to $5.8 \%$ in $2008 / 2009$.

Erectile dysfunction (ED) is a common but often neglected complication of diabetes which can adversely affect peoples quality of life ${ }^{1}$. Early studies indicated that among elderly men, ED can be the presenting symptoms of diabetes and the prevalence of undiagnosed diabetes is higher among ${ }^{2}$ people with $\mathrm{ED}^{3}$. Similar to DM, ED prevalence rates increase with age $^{4-5}$.

ED affects an estimated $34 \%-45 \%$ of men with DM worldwide $^{6}$ with individual studies reporting ED prevalence rates ranging from $20 \%$ to $90 \%{ }^{7}$. ED affects $>20$ million American men ${ }^{8}$.

Studies suggested the consumption of fruit and vegetable has beneficial effects on cardiovascular disease(CVD). ED and CVD are thought to be closely intertwined disease processes $^{9-10-11}$ and share common risk factors with diabetes. Therefore, it is of interest to know what the association is between ED and the consumption of fruits and vegetables among diabetic men.

Two studies of Esposito et al. ${ }^{12-13}$ observed a protective effect for ED in relation to Mediterranean diet, which is rich in fruits and vegetables.

\section{Aim}

Our study evaluates the association between fruit/vegetable consumption and ED among Albanian men with DM.This is the first study, in our country exploring such association directly at population level.

\section{Method}

Data from the Public Health Agency of Albania's 2014

Survey on Living with Chronic Diseases in Albania-Diabetes Components, were used in this study. This survey, conducted by Statistics Albania, interviewed a nationally representative sample of 475 responders (aged 20 years or older, with an average age of 64 years) with self - reported diabetes diagnosed by a health professional. The survey response rate was $81.4 \%$.

ED was identified on the basis of the survey question: "Have you ever had any of the following conditions: ED?" In the survey, the daily consumption of fruits/vegetables was estimated on the basis of a series of questions: 1)" How often do you usually eat fruits?"; 2) "How often do you usually eat vegetables"?. It is assumed that consumption of

fruits/vegetables on time per day is approximately equal to one serving.

The total daily consumption of fruits/vegetables was derived from these questions with possible responses of:"less than 3 times/per day "; " 3 to 5 times/ per day "; "more than 5 times/per day". The association between fruit/vegetable consumption and erectile dysfunction was examined using logistic regression. Bootstrap procedure was used to estimate sample distribution and calculate Confidence Intervals(CI).

\section{Analysis}

Data analysis was conducted using Statistical Analysis System Enterprise Guide (SAS-EG).Socio demographic characteristics, health status, lifestyle behaviours, and key diabetes complications were calculated and compared 


\section{International Journal of Science and Research (IJSR) \\ ISSN (Online): 2319-7064 \\ Index Copernicus Value (2013): 6.14 | Impact Factor (2015): 6.391}

between persons with erectile dysfunction and persons without erectile dysfunction. We combined underweight, body mass index $(\mathrm{BMI})<18.5 \mathrm{~kg} / \mathrm{m}^{2}$ and normal weight 18.5 and $<25 \mathrm{~kg} / \mathrm{m}^{2}$, because of the small number of people who were underweight. Rates and 95\% CI were calculated and weighted to represent the Albanian population. The association between fruit/vegetable consumption and erectile dysfunction was examined with logistic regression analyses while adjusting for potential confounding factors, including duration of diabetes, type of diabetes, CVD, and physical activity.

\section{Results}

This study includes a total of 475 men, with an average age of 60 years (range 20-70 years). Most of patients living in urban setting, married, or living common -law, with a lower income level and a lower education level as is most of people level in Albania. A total of 205 cased of ED were identified. Overall $89.1 \%$ of patients reported having -type 2 diabetes, $7.3 \%$ reported having type 1 diabetes, and $3.6 \%$ of responders were unclassified (did not know, not stated, not applicable, and other). Nearly $82 \%$ of responders were overweight or obese. Overall 26.8\% (95\% CL) of responders reported having ED, with rates steadily increasing with age and duration of living with the disease. Higher proportions of patients with ED reported poor fair health, long duration of diabetes ,obesity, physical inactivity, formerly smoking status, divorced and not consuming enough fruit and vegetables( $<3$ servings/day). Patients with ED reported significantly higher rates of hypertension, calculation problems, CVD, kidney disease, and nerve damage. An inverse association was found between ED and fruit/vegetable consumption. The rate of ED decreases by 13 $\%$ with each increase of 1 serving of fruit/vegetable per day.

\section{Comment}

One U.S. study reported that ED affected $>50 \%$ of men aged 40-70 years, diabetic or not. Our findings confirm that ED is a common problem in men with diabetes, in Albania. More than 1 in 5 Albanian men are with diabetes, aged 22 years and older, reported that they had consulted a physician regarding ED. After adjustment of age, ED is significantly associated with mental status, education attainment level, smoking, fruit vegetables consumption, BMI, and the presence of other diabetes complications. Specifically, the likelihood to have ED is doubled among smokers. Smoking is an established risk factor for $\mathrm{ED}^{14}$.

The consumption of fruits and vegetables is inversely associated significantly with ED. The association is independent from other factors. Our findings show a clear dose-response relationship between number of fruit/vegetable servings and risk of ED.

The benefit of fruit and vegetable consumption could be because of that they provide great nutrients, such as vitamins, amino acids, antioxidants, and citrulline, which could raise nitric oxide levels in human body to raise erectile function among men ${ }^{15}$. Diabetic patients are susceptible to ED, and erectile function could be more sensitive to dietary effects. Compared with the aforementioned 3 studies after controlling of confounding factors, the effect of fruit/vegetable consumption on ED among diabetic men in the present study seems unique in the context of health promotion.

\section{Conclusion}

The occurrence of ED among diabetic men in Albania is fairly common. ED is inversely associated significantly with the consumption of fruits and vegetables in the diabetic population. The inverse effect increases with the number of fruit/vegetable servings each day. In the addition, ED is also associated with mental status, cigarette smoking, elevated BMI, and other diabetic complications. Our finding suggest that adopting a healthy diet, healthy lifestyle, and reducing or controlling body weight would reduce the risk of developing ED.

\section{References}

[1] Lewis RW. Epidemiology of erectile dysfunction. UrolClin North Am. 2001; 28: 209-216.

[2] Deutsch S, Sherman L. Previously unrecognized diabetes mellitus in sexually impotent men. JAMA. 1980; 244: 2430-2432.

[3] Rosen RC, Fisher WA, Eardley I, et al. The multinational Men's Attitudes to Life Events and Sexuality (MALES) study: I. Prevalence of erectile dysfunction and related health concernes in the general population. Curr Med Res Opin. 2004; 20: 607-617.

[4] Rubin A, Babbott D. Impotence and diabetes mellitus. JAMA. 1958; 168: 498-500.

[5] Feldman HA, Goldstein I, Hatzichristou DG, et al. Impotence and its medical and psychological correlates: results of the Massachusetts male aging study. J Urol. 1994; 151: 54-61.

[6] Brock G, Bebb R, Harper W. Erectile Dysfunction. Clinical Practice Guidelines for the Prevention and Management of Diabetes in Canada, 2008. Canadian Diabetes Association 2008 Canadian Journal of Diabetes, 2008; 32 (S1): s147-s 149.

[7] Ma RC, So WY, Yang X, et al. Erectile dysfunction predicts coronary heart disease in type 2 diabetes. $\mathrm{J}$ Am CollCardiol. 2008; 51: 2045- 2050.

[8] Chu NV, Edelman SV, Erectile dysfunction and diabetes. CurrDiab Rep. 2002; 2: 60-66.

[9] Menezes A, Artham S, Lavie CJ, et al. Erectile dysfunction and cardiovascular disease. Postgrad Med. 2011; 123: 7-16.

[10] Billups KL. Sexual dysfunction and cardiovascular disease: integrative concepts and strategies. Am J Cardiol. 2005; 96(12B); 57M-61M.

[11] Feldman HA, Johannes CM, Derby CA, et al. Erectile dysfunction and coronary risk factors prospective results from the Massachuusetts male aging study. Prev Med. 2000; 30: 328-338.

[12] Esposito K, Giuliano F, De SioM,et al. Dietary factors in erectile dysfunction. Int J Impot Res. 2006; 18: 370 374. 
[13] Esposito K, Ciotola M, Giuliano F, et al. Mediterranean diet improving erectile function in subjects with metabolic syndrome. Int J Impot Res. 2006; 18: 405410.

[14] Polsky JY, Aronson KJ, Heaton AJ, et al. Smoking and other lifestyle factors in relation to erectile dysfunction. BJU Int. 2005; 96: 1355-1359.

[15] Drewes SE, George J, Khan F. Recent findings on natural products with erectile-dysfunction activity. Phytochemistry. 2003; 62: 1019-1025. 